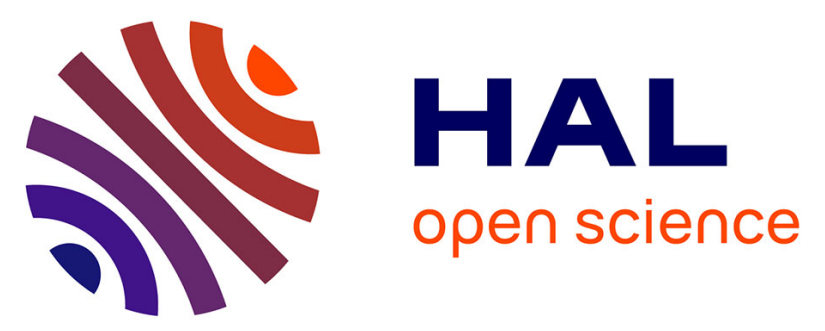

\title{
Ultra-light extensometer for the assessment of the mechanical properties of the human skin in vivo
}

Emmanuelle Jacquet, Sylvain Joly, Jérôme Chambert, Khansa Rekik, Patrick

Sandoz

\section{- To cite this version:}

Emmanuelle Jacquet, Sylvain Joly, Jérôme Chambert, Khansa Rekik, Patrick Sandoz. Ultra-light extensometer for the assessment of the mechanical properties of the human skin in vivo. Skin Research and Technology, 2017, 10.1111/srt.12367 . hal-01501629

\section{HAL Id: hal-01501629 \\ https://hal.science/hal-01501629}

Submitted on 4 Apr 2017

HAL is a multi-disciplinary open access archive for the deposit and dissemination of scientific research documents, whether they are published or not. The documents may come from teaching and research institutions in France or abroad, or from public or private research centers.
L'archive ouverte pluridisciplinaire HAL, est destinée au dépôt et à la diffusion de documents scientifiques de niveau recherche, publiés ou non, émanant des établissements d'enseignement et de recherche français ou étrangers, des laboratoires publics ou privés. 


\title{
Ultra-light extensometer for the assessment of the mechanical properties of the human skin in-vivo
}

\author{
Emmanuelle Jacquet, Sylvain Joly, Jérôme Chambert, Khansa Rekik, \\ Patrick Sandoz \\ Univ. Bourgogne Franche-Comté, FEMTO-ST Institute, CNRS/UFC/ENSMM/UTBM
}

\author{
Corresponding author: \\ Dr. Emmanuelle Jacquet, \\ email: emmanuelle.jacquet@univ-fcomte.fr \\ Phone: +33381666 019 \\ Fax: +33 381666700 \\ Univ. Bourgogne Franche-Comté, \\ FEMTO-ST Institute, \\ CNRS/UFC/ENSMM/UTBM, \\ Department of Applied Mechanics, \\ 24, rue de l'Epitaphe, \\ 25000 Besançon, France
}

Number of pages: 21

Number of table: 1

\begin{abstract}
Background/Purpose: This paper aims to present an ultra-light extensometer device dedicated to the mechanical characterization of the human skin in vivo.

Methods: The device developed was conceived to be non invasive, to work without any stand and to perform various uniaxial tensile tests with either effort or displacement control. We also use specific guarding tabs to make in vivo extension tests analogous to traction tests.

Results: Force-displacement curves are derived from the data provided by the device's sensors. The latter are converted into stress-strain curves thanks to complementary measurements of the skin thickness. We present typical experimental data and results that demonstrate the device ability to built stress-strain curves characteristic of the human skin behavior. An additional imaging unit records a sequence of images of the solicited skin area for further calculations of the displacement fields by digital image correlation.

Conclusion: The analysis of the displacement and deformation fields validates the guarding tab efficiency and the capacity of the device to characterize the mechanical behavior of the human skin in vivo.
\end{abstract}

Keywords: Extensometer, Skin in vivo, Mechanical properties, Standalone instrument, Full field measurement, Digital Image Correlation. 


\section{INTRODUCTION}

The ability to predict the in vivo response of the human skin to a mechanical loading is crucial to various domains, either clinical (sutures techniques, follow-up of skin pathologies or tissue bio-engineering) or related to body care research (air-bag design or efficiency testing of cosmetic products). For this purpose, surgeons, biologists, doctors and engineers have been trying for a long time to evaluate the mechanical properties of this heterogeneous, viscoelastic, anisotropic, non-linear and adhesive material.

Basically, the mechanical behavior of the skin can be tackled by two categories of tests; i.e. perpendicularly to the skin surface (succion [1], contact indentation [2] [3] or contactless indentation [4]) or in the plane of the skin surface (torsion [5] or extension [6]). The extension test consists in moving two pads attached to the skin surface and in measuring the force induced by the pad displacements.

Many extensometers for the in vivo skin have been designed in association with a stand. The first of them [7] served as a basis for several more recent ones $[8,9,10,11,12]$. Both Evans and Boyer combined mechanical and optical measurements in their tensile devices $[13,14]$. A three-pad extensometer was also designed with the aim to protect the measuring zone from peripheral forces [15]. In all those instruments hold by a stand, the parasitic forces due to interactions between the tested skin and the experimental device have to be kept sufficiently small to be neglected or measured to be taken into account during measurement analysis. This point is critical since movements due to either breathing or blood flow cannot be canceled.

To solve this problem, various hand-portable devices operating directly on a free skin were developed to be insensitive to small body movement disturbances $[16,17,18]$. Those extensometers are suited for application on almost any body zone compatible with the device size.

The rigid attachment of the extensometer pads to the skin is required by all the devices mentioned above. No fully satisfactory solution does exist and 
liquid (cyanocrylate) or double-sided adhesive tapes are the most commonly used means.

The skin response to any mechanical loading depends on diverse environmental and instrumental parameters that have to be monitored. Even in best measurement conditions, huge differences are observed in the human-skin mechanicalcharacteristics as reported in literature. The human skin is indeed not made of a standardized material with constant specifications. It corresponds rather to a generic material of which mechanical properties vary between individuals as well as between body zones [12]. This variability raises a methodological challenge since averaged values cannot be used to predict the skin response to a specific solicitation.

With the aim to shift toward a personalized medicine, the actual properties of the skin to be treated have to be measured and suitable instruments are required. This paper presents an ultra-light standalone device developed for that purpose and reports on its ability to characterize the mechanical response of the human skin in vivo to diverse mechanical tests. The extensometer design and control are described and its functioning is validated from reference measures. Its ability to assess the mechanical properties of the human skin in vivo are illustrated by typical results. Finally the device performances are analyzed and discussed.

\section{Extensometer development, control and validation}

\subsection{Aim and design}

The extensometer was designed to fulfill the following specifications :

- usable in most of the human sites in vivo,

- light enough to work as a standalone instrument,

- easy to use and consistent with standards and biomedical requirements,

- performing extension tests with either force or displacement control (loading and unloading), 


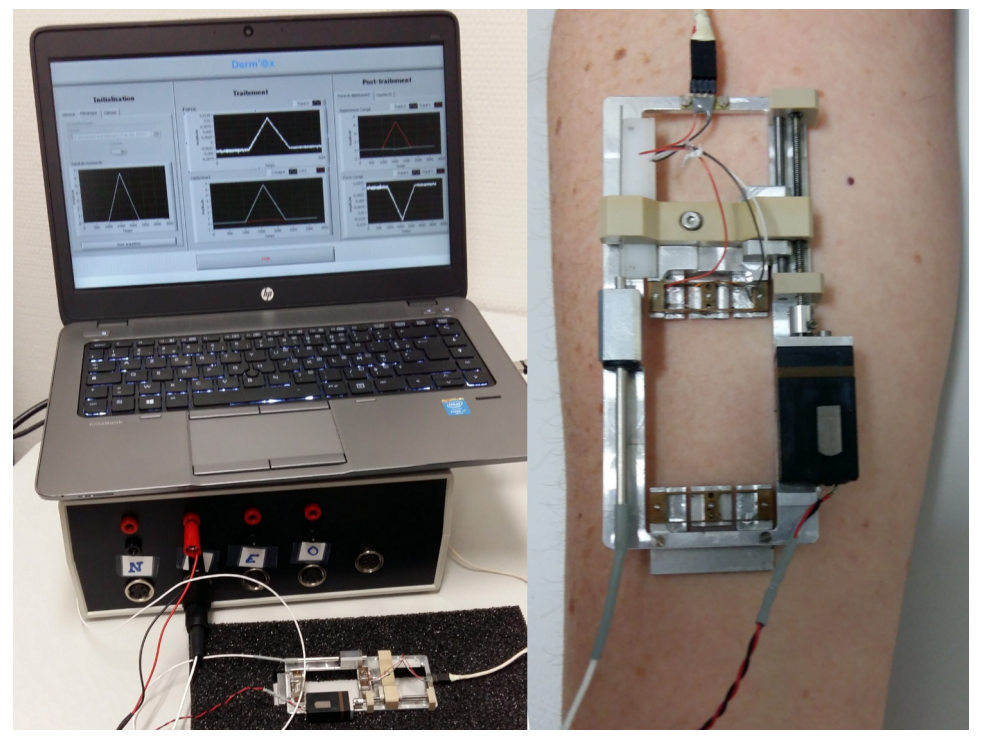

Figure 1: Left: Electronic and mechanical parts of the device. Right: Setup in measuring situation on a forearm.

- permitting large quasi-static displacements as well as low-frequency dynamic solicitations on the human skin in vivo,

- keeping the tested skin area visible to permit simultaneous image acquisition.

The whole experimental setup is shown in figure 1 whereas the details of the extensometer are shown in figure 2. The specifications of the device built are summarized in table 1. A speckle pattern is applied on the skin for the sake of accurate local-motion detection by means of digital image processing (cf. 2.3).

\subsection{Mechanical part}

The device is made of a rectangular frame $\left(100 \times 40 \times 6 \mathrm{~mm}^{3}\right)$ at which are fixed an electric motor, two guiding pillars and two sets of complementary pads: one static, one mobile (cf. Fig. 2). The mobile set of pads is held by a traverse beam translated by the motor by means of a screw-nut system. Each set of pads is made of a measuring pad $\left(8 \times 8 \mathrm{~mm}^{2}\right.$ called central pad $)$ surrounded 


\begin{tabular}{|c|c|c|}
\hline \multirow{3}{*}{ General specifications } & \multicolumn{2}{|l|}{ Central Pads and guarding pads } \\
\hline & \multicolumn{2}{|l|}{ Force or displacement control } \\
\hline & \multicolumn{2}{|l|}{ Bonding pads } \\
\hline \multirow{9}{*}{ Technical specifications } & Dimensions with motor & $100 \times 40 \times 10 \mathrm{~mm}^{3}$ \\
\hline & weight & $36 \mathrm{~g}$ \\
\hline & Observable area & $<45 \times 24 \mathrm{~mm}^{2}$ \\
\hline & Effort sensor & $5 \mathrm{~N}$ \\
\hline & Effort accuracy & $5 \mathrm{mN}$ \\
\hline & Maximal displacement & $15 \mathrm{~mm}$ \\
\hline & Maximal strain & 0,5 \\
\hline & Maximal displacement speed & $1 \mathrm{~mm} / \mathrm{s}$ \\
\hline & Sampling rate & 100 samples/s \\
\hline
\end{tabular}

Table 1: Specifications of the device. 


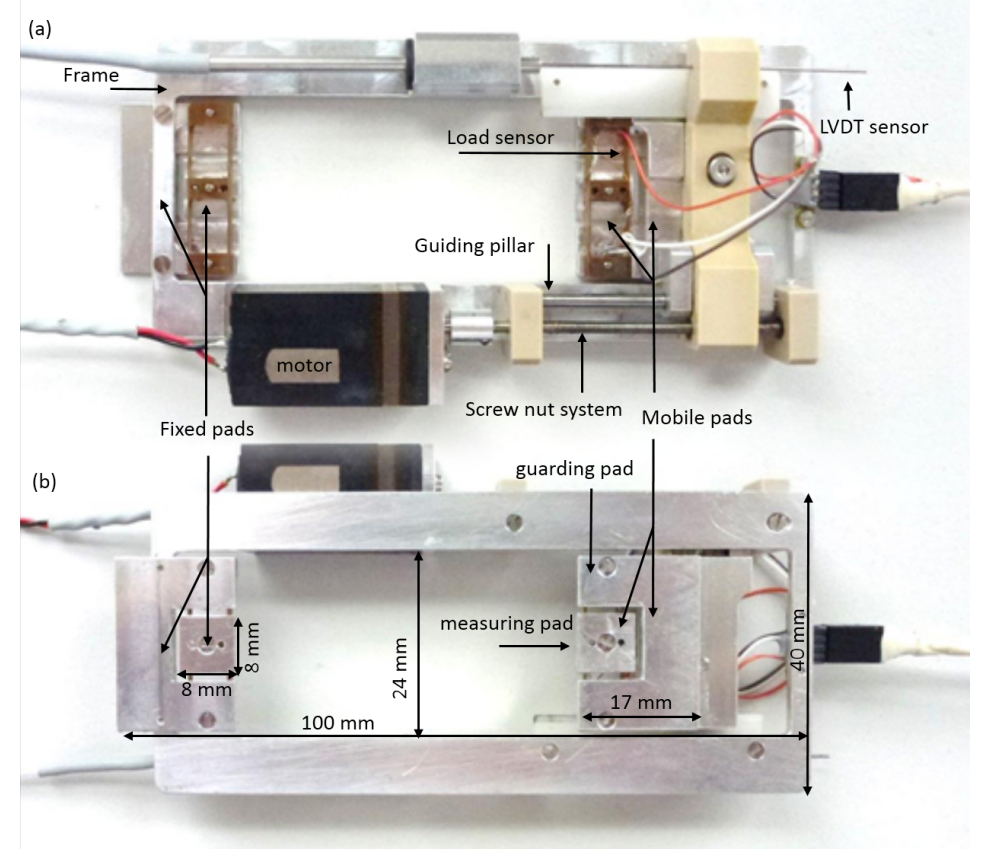

Figure 2: Top (a) and bottom (b) views of the extensometer.

by a "U-shaped" pad $\left(24 \times 17 \mathrm{~mm}^{2}\right.$ called guarding pad $)$. The guarding pads protect the measuring zone from lateral and peripheral forces by shifting the border between the solicited and unsolicited zones towards the outside [15]. The central and guarding pads are moved together but the force is measured from the central pad only; i.e. one third of the solicited width. The ratio between length and width of the force-measurement region is larger than 3 . The force sensor is made of a specific bronze-berrylium sensor equipped with strain gauges stuck on the sensor where the strain due to flexion is maximal. The device is supplemented by a LVDT-type displacement sensor providing the mobile pad position.

\subsection{Imaging part}

A vision system is associated to the extensometer to allow the measurement of the field of displacements on the solicited area during the mechanical test by means of digital image correlation (DIC). The camera is hold by a six degrees 
of freedom stand independent of the extensometer and allowing to focus on the tested skin area. Images are recorded at a $30 \mathrm{fps}$ rate and post-processed using Matlab.

Since the extensometer is fixed on the skin through its four pads of which two are moving, some slight displacements of the device on the skin occur during mechanical tests in addition to slight body movements. To avoid biases due to these disturbances, the field of observation of the vision system is chosen large enough to make the whole device visible. All images are registered within a single $(X, Y)$ coordinate reference from the positions of the pads that provide also a size reference to convert pixels into actual sizes without further calibration.

A random pattern is applied onto the skin using a water-based paint with a brush as required by the DIC method used [19]. The digital method consists in comparing the current image to the initial one to retrieve the averaged displacement values of sub-images of $32 \times 32$ pixels. The software looks for the displacement value that minimizes the correlation function built from the products of the functions describing both the reference and current images. The method is applied to the whole sequence of images to provide the fields of displacements for the entire duration of the mechanical test.

\subsection{Electronic control}

An electronic controller based on a microcontroller (Arduino Mega 2560) was developed to drive the extensometer motor and to acquire the data from the embedded sensors.Two Arduino shields based on H-bridge circuits command the motors by means of a Pulse-Width-Modulate (PWM) signal. A Linear Variable Displacement Transducer (LVDT) provides a tension proportional to the position of the pad that is detected thanks to an adaptation card (EL35C-Analog by Singer Instrument). A supplementary electronic card (based on INA2126) shifts the LVDT signal in always-positive tensions as required by the microcontroller. The signal provided by the strain gauge is amplified by a conditioning card (based on LM358). The user accesses the microcontroller parameters by means of a software interface developed under Labview. 


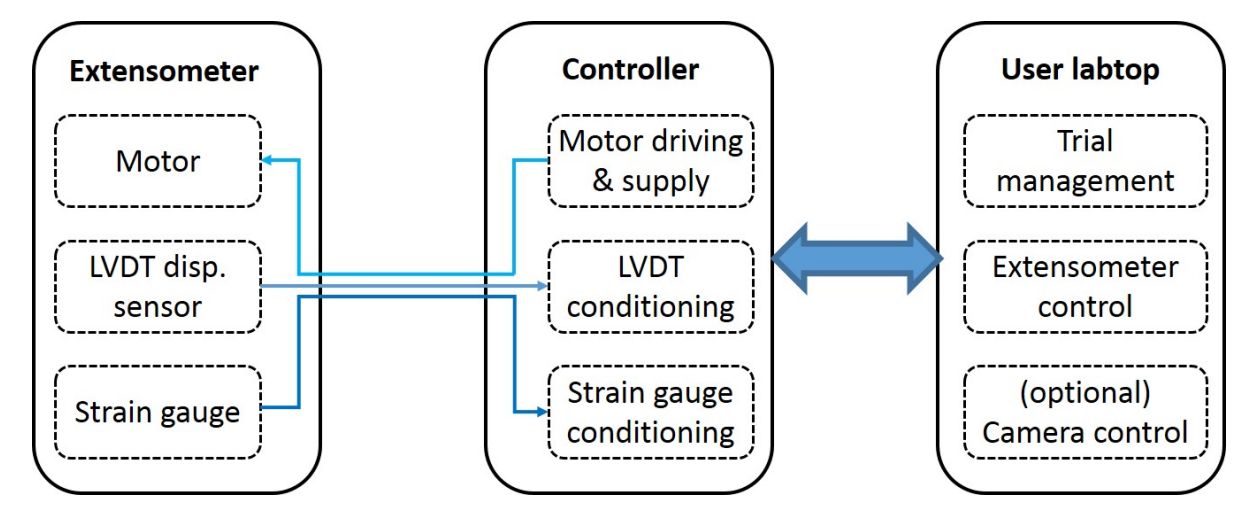

Figure 3: Extensometer control.

\subsection{User assistance interface}

Two complementary software applications were developed under Labview. One is dedicated to the easy generation of motor driving command files. The latter are built only once and available to proceed to identical tests on different subjects or different skin areas. Any kind of test is allowed (quasi-static, harmonic, ...) as long as within the device specifications (speed $<1 \mathrm{~mm} / \mathrm{s}$, displacement $<15 \mathrm{~mm}$ and force $<5 \mathrm{~N}$ ) without other limitations.

The second software application is dedicated to the overall control of the device and experiments (Fig. 3). It is made of three independent and complementary blocks: extensometer control, (optional) camera control, documentation of the ongoing trial. The extensometer can be controlled either automatically by means of a driving command file or manually by means of virtual potentiometers. The displacements of the mobile pad are servo-controlled either in displacement or in force. The camera control block aims to the driving of an optional camera. It allows the recording of a set of images of the tested skin area synchronously with the motion applied to the pad. The images obtained are stored in a folder attached to the ongoing trial for further digital image processing.

Finally the trial documentation block allows the storage of the data relevant to the current experiment. Predefined fields are proposed for generic data (age, 
sex, weight,...$)$ whereas additional fields can be created and filled according to the user needs. A trial report is automatically generated at the end of every trial thus making the all data available for further consultation or analysis.

\subsection{Validation of the instrument}

Specific experiments were carried out to validate the correct functioning of the extensometer by comparing the force and displacement values provided by the strain gauges and the LVDT sensor to reference ones. The correct functioning of the instrument was also checked by comparing the results provided by our device on a thin sample of elastomer to those obtained from a traction machine (BOSE Electroforce 3200) on the same material. Despite the large area of the tested sample (analog to actual experiments on the skin), the agreement observed validated the correct functioning of our extensometer.

A standalone device is easy to use and does not limit the patient's movements. Force and displacement sensors are embedded and follow naturally the

global motion. At contrary the camera is fixed and detect rigid-body patient's displacements. The correct registration of the sequences of images was validated by the successful comparison of the data provided by the LVDT sensor during a loading-unloading test with those obtained by DIC.

The low device mass in regard to its surface of contact (pads plus frame) results in applying a very weak pressure onto the tested skin. The induced disturbances are therefore considered as negligible, thus allowing the use of the device without a stand provided that patient movements remain sufficiently small and slow.

\section{Experiments on the human skin in vivo}

\subsection{Experimental protocol}

\subsubsection{Ethical aspects}

Tests on living tissues are submitted to permission by the ethical board of the hospital as well as to the subject's consent once the person was fully 
informed regarding the test objectives and experimental protocol. To avoid any risk to the patient's safety, a risk table was established. The major element able to produce damages was identified as an uncontrolled extension applied to the skin. This possibility was discarded by means of an emergency stop button that triggers the instantaneous release of the loading (or unloading) forces applied to the skin. Furthermore the interface screen plots in real-time the measured values of both the force and the displacement thus allowing an additional monitoring of the ongoing test by the operator.

\subsubsection{Mode of operation}

Some test preparation is necessary on both patient and operator sides and the full sequence of test operation takes place as indicated below:

- $20 \mathrm{mn}$ acclimatisation period to ensure constant environmental conditions.

- Speckle pattern realization.

- Test documentation on the software interface (subject identifier, age, sex, body zone, test orientation, etc.).

- Loading of the test command file, either already existing or just created.

- Sticking of the extensometer on the patient's skin (hardening in 20-30 s) and camera focusing adjustment.

- Launching of simultaneous test and image recording. Data are stored in a specific directory and summarized in an automatic test report.

- Extensometer removal and cleaning.

- Data available for DIC computations and result analysis.

\subsection{Typical results}

A huge diversity of tests with either force or displacement control are allowed by the device. Figure 4 presents three typical results obtained with displacement control. The $1^{\text {st }}$ row presents the displacements applied to the skin as measured 

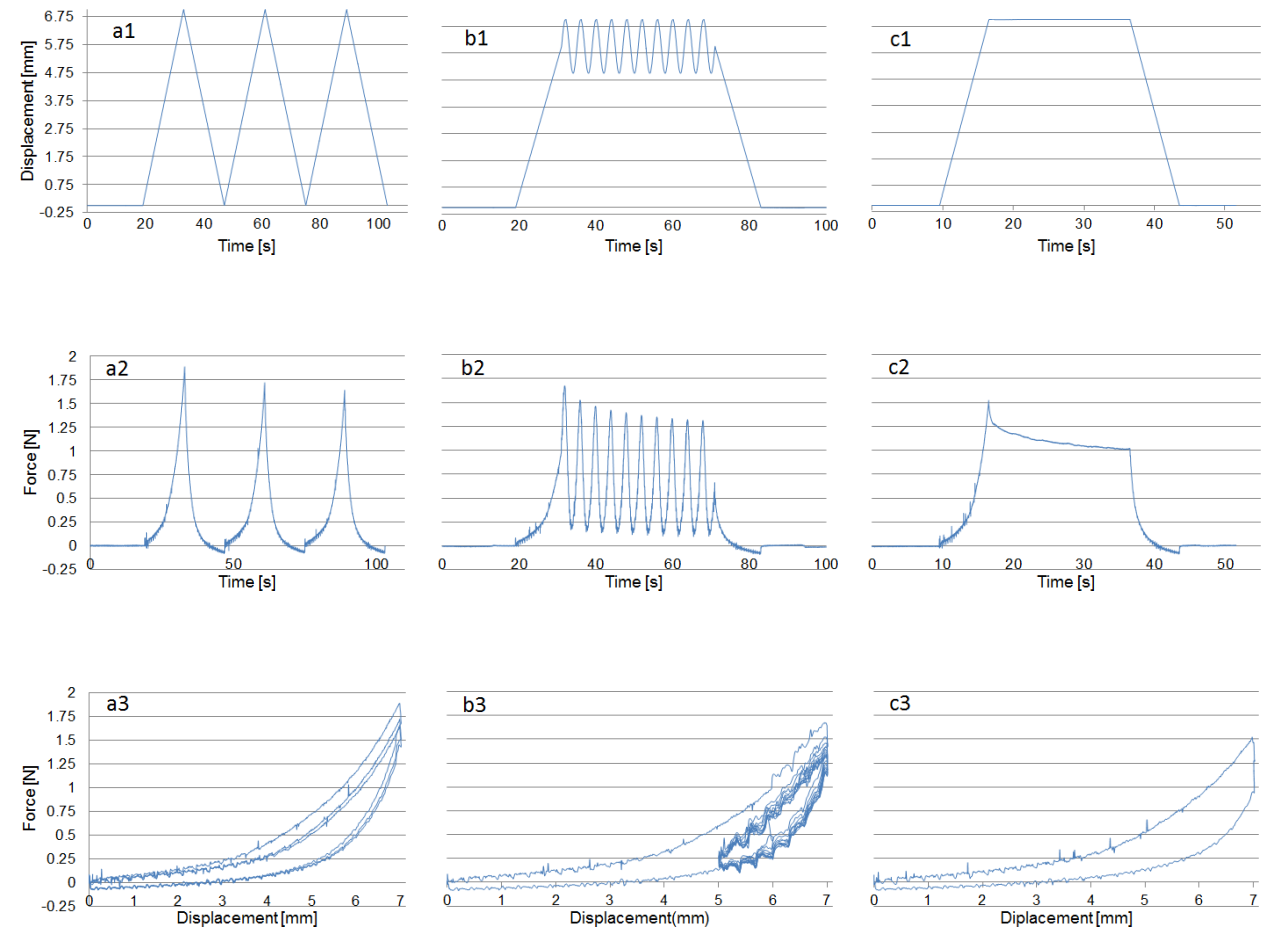

Figure 4: Typical results: Column a): three successive loadings-unloadings, Column b): harmonic loading after a pre-load; Column c): loading-holding-unloading. $1^{\text {st }}$ row: Displacement returned by the LVDT. $2^{\text {nd }}$ row: measured force; $3^{\text {rd }}$ row: force-displacement curves.

by the LVDT whereas the $2^{\text {nd }}$ row plots the measured forces. The $3^{\text {rd }}$ row corresponds to the force-displacement curves obtained. Column (a) corresponds to a sequence of three consecutive loading-unloading. By superimposed a harmonic load to a static pre-load, column (b) gives some ideas on the dynamic behavior of the skin. Finally, column (c) corresponds to a loading-holding-unloading sequence exhibiting viscoelastic properties.

The displacement sensor response $\left(1^{\text {st }}\right.$ row) agrees with the input control command send to the device up to the maximal speed of $1 \mathrm{~mm} / \mathrm{s}$. The force response ( $2^{\text {nd }}$ row) is highly non-linear as typically expected from a hyperelastic material. In repeated (a1) and harmonic (b1) loadings, the maximal force value decreases with the first cycles to stabilize once preconditioning is obtained (a2, 
b2). The hysteresis curves (a3, b3) are representative for the material dissipative character. During the holding step the measured force presents an exponentially decreasing shape as expected (c2). The holding time of $20 \mathrm{~s}$ is nevertheless too short to observe the force stabilization.

The recorded data are available for further analysis and material behavior modeling using either hyperelastic or viscoelastic models.

\subsection{Performance analysis}

\subsubsection{Ability to report on the skin behavior}

The simultaneous measurement of forces and displacements can be analyzed as stress-strain curves since the test can be considered as a uniaxial traction test (cf. 3.3.2 and 3.3.3). The proposed device allows the demonstration of the non-linear elastic behavior of the tested skin and the parameters of an isotropic hyperelastic model can be identified from such uniaxial extension tests. The analysis of the unloading and holding phases requires the introduction of viscosity terms in the behavior models.

The use of the complementary imaging unit provides the field of displacements over the entire field of view as represented in Fig. 5. We observe that the deformed zone extends beyond the area between the measuring pads and even beyond the guarding pads. For a given X-position, the X-displacement parallel to the tensile axis is uniform over almost the whole transverse direction. The deformation field $\epsilon_{x x}$ is almost constant over the all area thus confirming that the $\mathrm{X}$-displacement is linearly distributed along the tensile axis. The field of transverse Y-displacements shows a shrinking of the zone between the pads as expected. The field of deformations $\epsilon_{y y}$ is also uniform and negative and the shear deformation field $\epsilon_{x y}$ is close to zero over the whole area. These results fit the behavior of a homogeneous material under uniaxial traction test.

Beyond these results, the analysis of digital imaging data reports on a possible heterogeneity in the skin behavior. Tests with holding phases can be compared with cutometry tests commonly used by dermatologists. The continuous and simultaneous measurement of both force and displacement allows to gather 


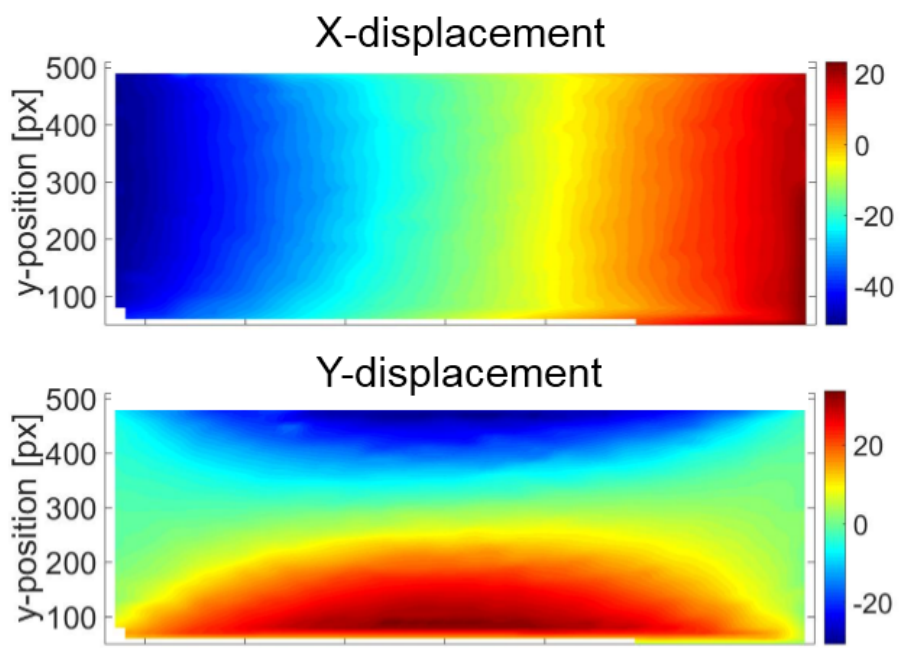

Strain $\varepsilon_{x x}$
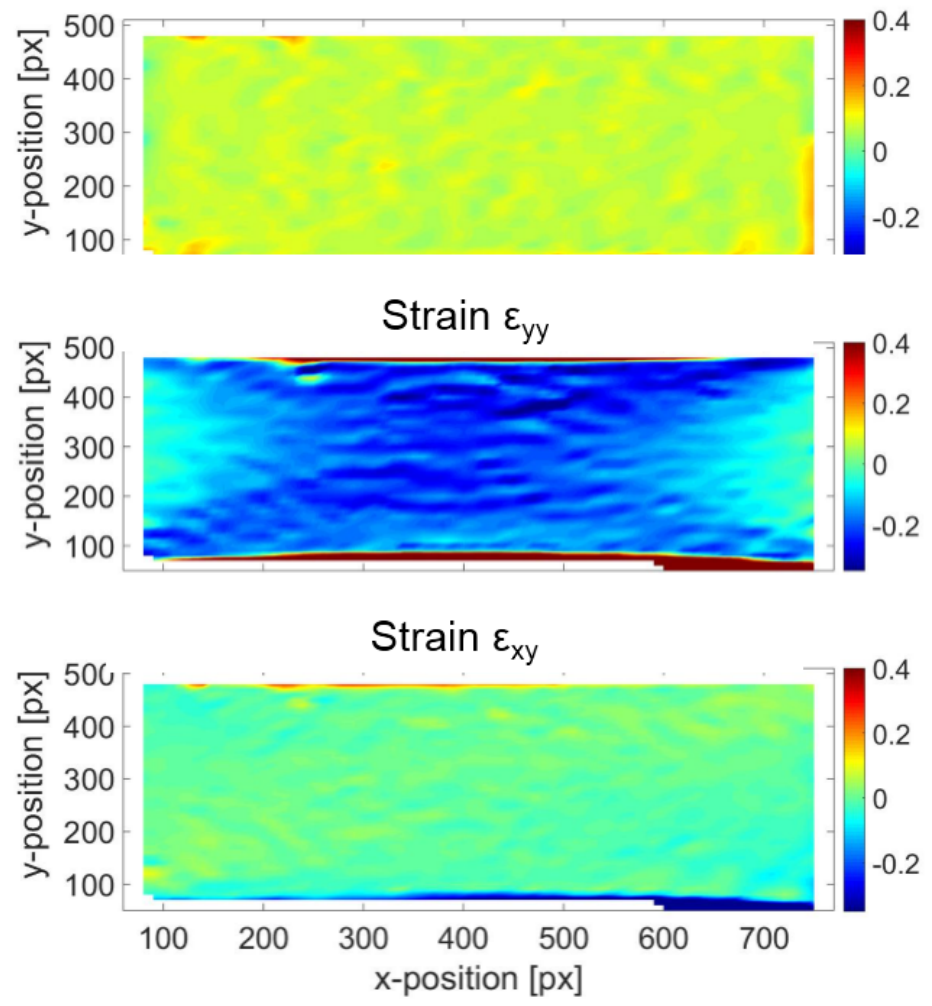

Figure 5: From top to bottom: Displacement fields following the tensile axis $\mathrm{X}$ and the transverse axis Y. Strain fields $\epsilon_{x x}, \epsilon_{y y}$ and $\epsilon_{x y}$ obtained from a test with displacement control. (Scales in pixels with $5.2 \mathrm{~mm}$ per 100 pixels). 
data necessary to address the skin viscosity. Finally harmonic loadings provide a means for identifying the intrinsic parameters of the material independently of its environment thanks to the decoupling of elasticity and viscosity parameters.

As reported in literature, mechanical measurements on the human skin present a high degree of variability. Even on the same zone of the same subject, results may differ as a function of time or conditions. The skin response to the same loading is not the same at the first, second or third cycle. Beyond the third cycle, we experimentally observed that a preconditioning is obtained and that the material response becomes reproducible.

\subsubsection{Efficiency of the guarding tabs}

The measurement zone is located between the measurement pads and, thanks to the guarding pads, the surrounding area is solicited in the same way. Disturbances due to distant unsolicited skin affect thus mostly the zone located between the guarding pads and are thus minimized on the central, actual measurement zone. The protective effect of the guarding pads can be assessed by comparing Fig. 6 obtained without sticking the guarding pads with Fig. 5. This comparison shows that the transverse uniformity is lost or significantly reduced for four represented variables amongst five. For Y-displacement, the transversal decrease of the displacement is smoother with the guarding tabs stuck. It should be noticed that the contour map of X-displacement is perpendicular to the tensile direction when the guarding pads are stuck (Fig. 5) whereas they are curved without the guarding pads (Fig. 6). This transverse uniformity is highlighted by Fig. 7 presenting the X-displacement over the all area between the pads whereas the zone between the measuring pads is marked by dotted lines. These figures confirm the efficiency of the guarding pads in minimizing the influence of the surrounding skin. Thanks to this protective effect, the extension test performed by the device can be considered as equivalent to a pure traction test with an excellent approximation.

At the level of the skin surface, the test produces an uniform longitudinal deformation on the skin located between the measuring pads. It is assumed that 


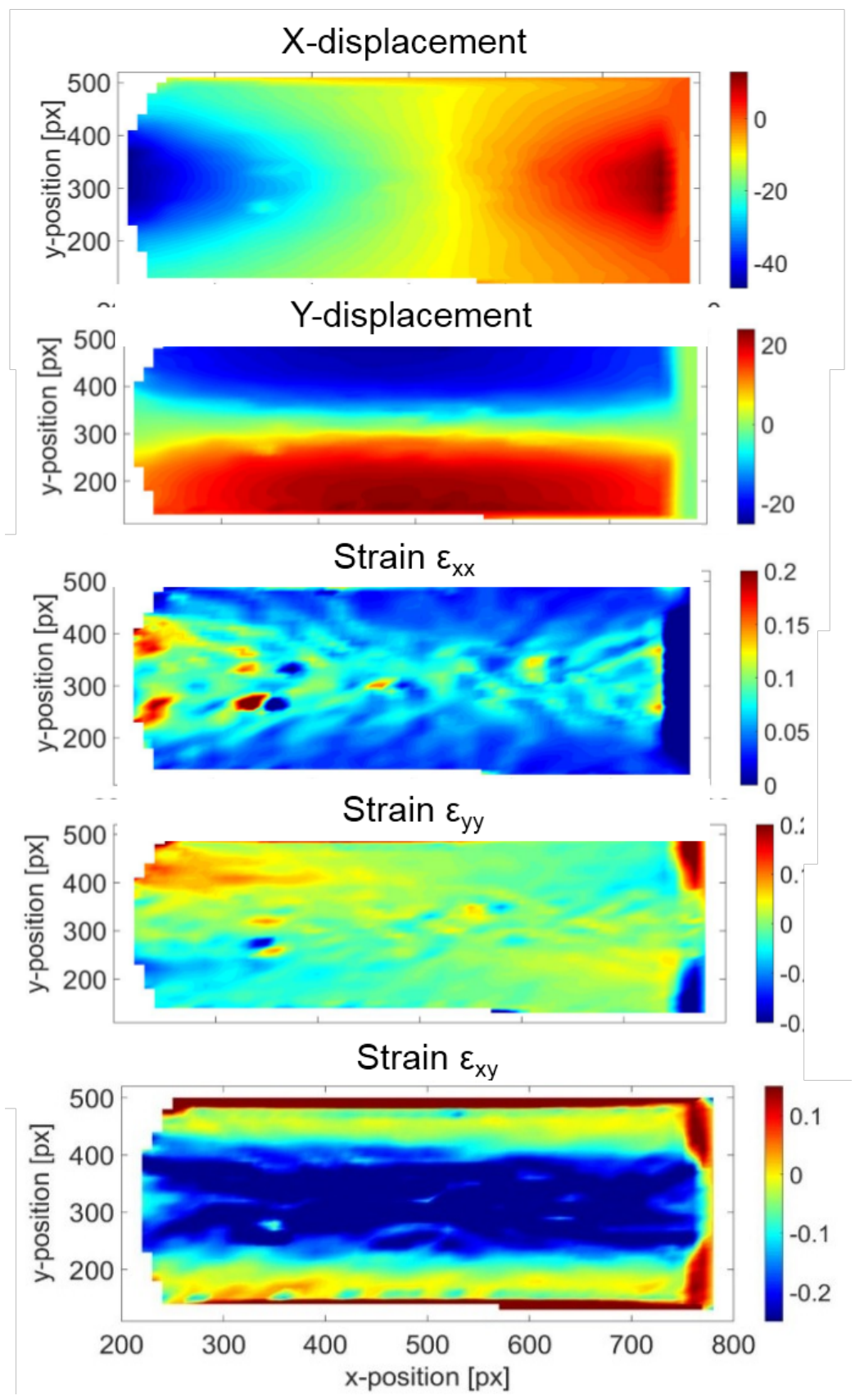

Figure 6: From top to bottom: Displacement fields following the tensile axis $\mathrm{X}$ and the transverse axis Y. Strain fields $\epsilon_{x x}, \epsilon_{y y}$ and $\epsilon_{x y}$ obtained from a test with displacement control and without guarding tabs. (Scales in pixels with $6.0 \mathrm{~mm}$ per 100 pixels). 


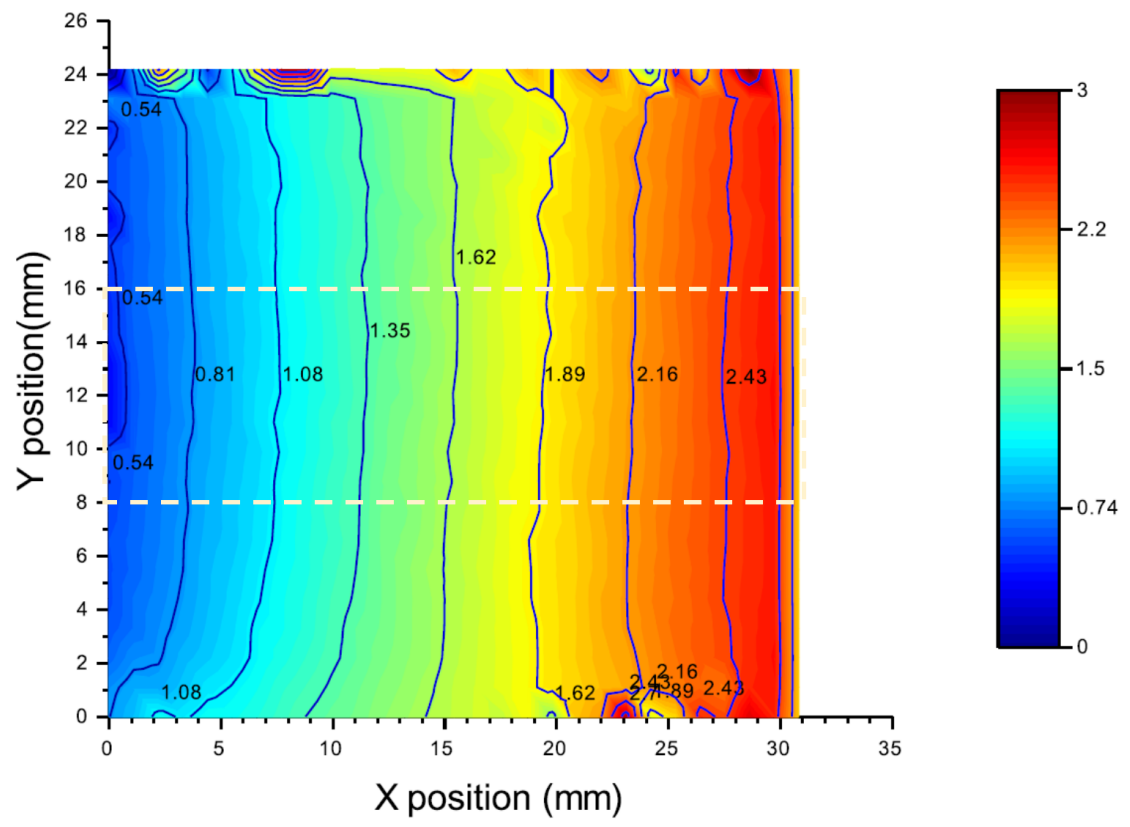

Figure 7: X-Displacement field following the tensile axis X obtained. (Displacement control with guarding pads. The zone between the dotted lines corresponds to the measuring pads.). 
the traction test applies homogeneously over the whole skin thickness $(\approx 1-2$

$\mathrm{mm}$ ). This hypothesis seems reasonable in regard to the distance between the pads and the deformation rates applied.

\subsubsection{Useful complementary measurements}

The skin thickness is measured after the extension test by ultrasound (Dermacup ATYS Medical, $25 \mathrm{MHz}$ probe). This allows the conversion of force data into stress data as applied to a tensile test-piece of width equal to the measuring pads one and with a thickness equal to the measured one.

The mechanical skin response to an extension test is also known to be sensitive to the degree of stratum corneum humidity. This parameter is kept relatively constant by respecting the biometrology tests requirements, especially the resting time in a room with controlled temperature and hygrometry.

\subsubsection{First in vivo results}

The device was applied to diverse series of tests in controlled conditions on the human skin in vivo, in particular on a cohort of 20 healthy people in our laboratory. Different anatomic zones were explored following different angular directions. The results obtained provide a wide source of data to analyze the human skin mechanical behavior. We notably document non-linear elasticity, anisotropy, preconditioning and initial tension of the human skin as well as its intra- and inter-individual variability. Fig. 8 illustrates the huge variability obtained on the force - displacement curves. In depth analysis and discussion of the results obtained on that cohort are presented in a dedicated paper [20] including the conversion of raw data into non-linear parameters characteristic of the human skin behavior.

\section{Discussion and conclusion}

A device performing uniaxial tensile tests with effort or displacement control has been presented in this paper. A displacement speed up to $1 \mathrm{~mm} / \mathrm{s}$ and a deformation up to $50 \%$ are permitted by the hardware. The pads are stuck 


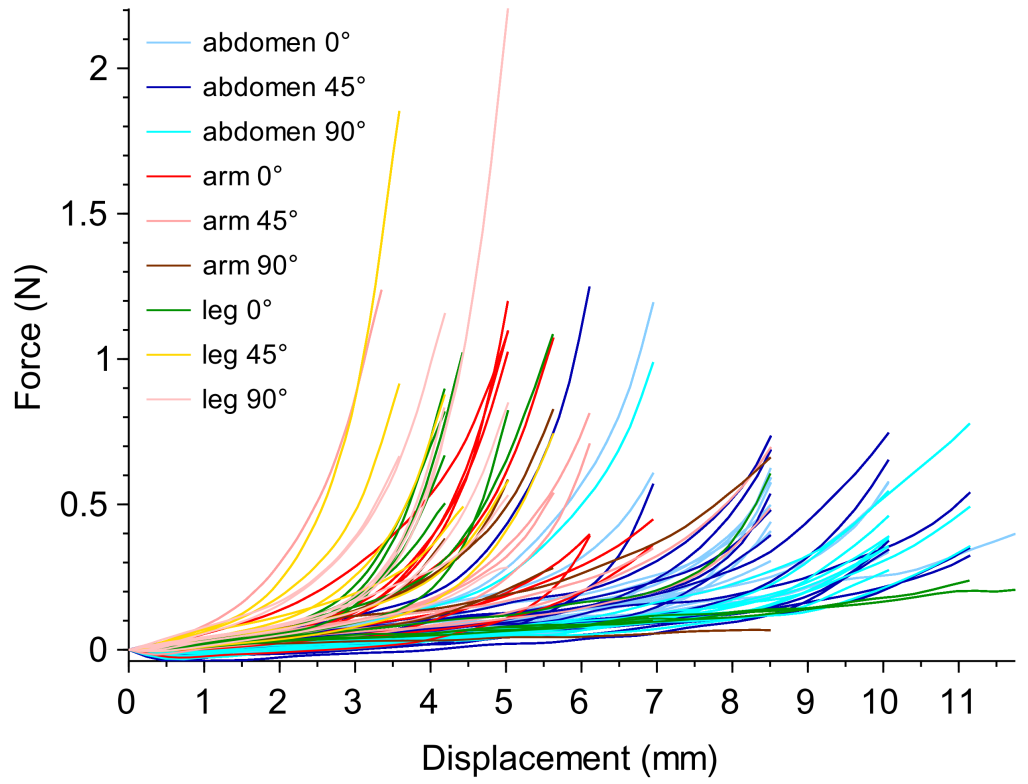

Figure 8: Illustration of the variability observed from a series of in vivo skin extension measurements. Tests on different people, different anatomic areas and different directions of solicitation. (Initial pad distance: $30 \mathrm{~mm}$ ). 
on the in vivo skin and the device lightness avoids the need for a stand. The advantage is that the patient can be either sitting, standing or laying and that his small movements do not disturb measurements without any control of the tangential efforts of the device onto the skin. This is especially important in regard to slight displacements induced by either breathing or blood flow. These specifications make thus the device operational on most body zones.

The guarding tabs minimize the effects of surrounding skin and the performed extension test can be considered as a traction test with a satisfactory approximation. Complementary thickness measurements allow the determination of stress-strain curves. In parallel, an independent imaging unit enables the image recording of the solicited skin area for the digital retrieval of the strain fields. The latter report on the heterogeneity of the solicited area. They also allow the identification of the intrinsic parameters of a material behavior model from the 2D strain distribution measured in response to an input control command in either moving-pad displacement or applied effort.

The software interface for generating input control command files offers a wide variety of solicitation types; either quasi-static or harmonic, with preload or holding steps. The material viscosity can thus be taken into account by identifying the parameters of a viscoelastic model. Successive tests can be oriented differently with respect to the Langer's lines to address the local skin

anisotropy. As a prospect, it is intended to develop a similar biaxial device to address the coupling between the axes of anisotropy of the material.

\section{Acknowledgements}

We are thankful to Eric Joseph for technical support and to the European Union for a FEDER grant from Région de Franche-Comté, France (n 36381).

\section{References}

[1] Diridollou S, Black D, Lagarde JM, Gall Y, Berson M, Vabre V, Patat F, Vaillant L. Sex- and site-dependent variations in the thickness and mechanical properties of human skin in vivo. Int J Cosmet Sci 2000;22:421-435. 
[2] Jachowicz J, McMullen R, Prettypaul D. Indentometric analysis of in vivo skin and comparison with artificial skin models. Skin Res Technol 2007;13:299-309.

[3] Boyer G, Laquièze L, Le Bot A, Laquièze S, Zahouani H. Dynamic indentation on human skin in vivo: ageing effects. Skin Res Technol 2009;15:55-67.

[4] Boyer G, Pailler Mattei C, Molimard J, Pericoi M, Laquieze S, Zahouani H. Non contact method for in vivo assessment of skin mechanical properties for assessing effect of ageing. Med Eng Phys 2012;34:172-178.

[5] De Rigal J. Hardware and Basic Principles of the Dermal Torque Meter in Bioengineering of the Skin, Skin Biomechanics, Volume V. (Elsner P, Berardesca E, Wilhelm KP, Maibach HI, eds., CRC Press) 2001, 63-76.

[6] Khatyr F, Imberdis C, Vescovo P, Varchon D, Lagarde JM. Model of the viscoelastic behaviour of skin in vivo and study of anisotropy. Skin Res Technol 2004;10:96-103.

[7] Thacker JG, Iachetta FA, Allaire PE, Edgerton MT, Rodeheaver GT, Edlich RF. In vivo extensometer for measurement of the biomechanical properties of human skin. Review Sci Instrum 1977;48:181-185.

[8] Manschot JFM, Brakkee AJM. The measurement and modelling of the mechanical properties of human skin in vivo - II. The model. J Biomech $1986 ; 19: 517-521$.

[9] Clark JA, Cheng JCY, Leung KS. Mechanical properties of normal skin and hypertrophic scars. Burns 1996;22:443-446.

[10] Vescovo P. Contribution à l'étude des propriétés mécaniques de la peau humaine. Mise au point d'une méthode pour l'expérimentation ex vivo. Conception et réalisation d'un dispositif d'essais en traction-compression in vivo. PhD thesis, University of Franche-Comté, France 2002. 
[11] Gahagnon S, Mofid Y, Josse G, Ossant F. Skin anisotropy in vivo and initial natural stress effect: A quantitative study using high-frequency static elastography. J Biomech 2012;45:2860-2865.

[12] Flynn C, Taberner A, Nielsen P. Measurement of the force-displacement response of in vivo human skin under a rich set of deformations. Med Eng Phys 2011;33:610-619.

[13] Evans SL. On the implementation of a wrinkling, hyperelastic membrane model for skin and other materials. Comput Method Biomech Biomed Eng 2009;12:319-332.

[14] Boyer G, Molimard J, Ben-Tkaya M, Zahouani H, Pericoi M, Avril S. Assessment of the in-plane biomechanical properties of human skin using a finite element model updating approach combined with an optical fullfield measurement on a new tensile device. J Mech Behav Biomed Mater 2013;27:273-282.

[15] Lim KH, Chew CM, Chen PCY, Jeyapalina S, Ho HN, Rappel JK, Lim BH. New extensometer to measure in vivo uniaxial mechanical properties of human skin. J Biomech 2008;41:931-936.

[16] Alexander H., Cook TH. Accounting for natural tension in the mechanical testing of human skin. J Investig Dermatol 1977;69:310-314.

[17] Jacquet E, Josse G, Khatyr F, Garcin C. A new experimental method for measuring skin's natural tension. Skin Res Technol 2008;14:1-7.

[18] Prevorovsky Z, Jacquet E, Placet V, Josse G, et al. Ultrasonic wave propagation and mechanical properties of human skin stretched in vivo in $2 n d$ International Conference on Mechanics of Biomaterials and Tissues, Kauai, Hawaii, 2007.

[19] Eberl C, Thompson R, Gianola D, Bundschuh S. Digital Image Correlation and tracking with MATLAB. Tech Rep 2010. 
[20] Jacquet E., Chambert J., Pauchot J., Sandoz P. Intra- and inter-individual variability in mechanical properties of human skin: Results from in vivo measurements with a portative extensometer. accepted in Skin Res Technol 